\title{
Prenatal dopamine and neonatal behavior and biochemistry
}

\author{
Tiffany Field $^{\mathrm{a}, \mathrm{b}, *}$, Miguel Diego ${ }^{\mathrm{a}}$, Maria Hernandez-Reif ${ }^{\mathrm{c}}$, Barbara Figueiredo $^{\mathrm{d}}$, \\ Osvelia Deeds ${ }^{\mathrm{a}}$, Angela Ascencio ${ }^{\mathrm{a}}$, Saul Schanberg ${ }^{\mathrm{e}}$, Cynthia Kuhn ${ }^{\mathrm{e}}$ \\ a Touch Research Institutes, University of Miami Medical School, United States \\ b Fielding Graduate University, United States \\ c University of Alabama, United States \\ d University of Minho, Portugal \\ e Duke University Medical School, United States
}

\section{A R T I C L E I N F O}

\section{Article history:}

Received 27 April 2007

Received in revised form 19 November 2007

Accepted 19 July 2008

\section{Keywords:}

Prenatal dopamine

Depression

\begin{abstract}
A B S T R A C T
Depressed pregnant women $(N=126)$ were divided into high and low prenatal maternal dopamine (HVA) groups based on a tertile split on their dopamine levels at 20 weeks gestation. The high versus the low dopamine group had lower Center for Epidemiological Studies-Depression Scale (CES-D) scores, higher norepinephrine levels at the 20-week gestational age visit and higher dopamine and serotonin levels at both the 20- and the 32 -week gestational age visits. The neonates of the mothers with high versus low prenatal dopamine levels also had higher dopamine and serotonin levels as well as lower cortisol levels. Finally, the neonates in the high dopamine group had better autonomic stability and excitability scores on the Brazelton Neonatal Behavior Assessment Scale. Thus, prenatal maternal dopamine levels appear to be negatively related to prenatal depression scores and positively related to neonatal dopamine and behavioral regulation, although these effects are confounded by elevated serotonin levels.
\end{abstract}

(c) 2008 Elsevier Inc. All rights reserved.

\section{Prenatal dopamine and depression}

Human and animal studies suggest a relationship between dopamine transmission and depression (Dailly, Chenu, Renard, \& Bourin, 2004 for a review). At least one study has reported lower dopamine levels in depressed pregnant women (Field et al., 2004). In that study, the newborns of depressed mothers also had low dopamine levels. Low dopamine has been implicated in lower orienting behavior in 2-week-old infants and more negative temperament in 2-month-old infants (Auerbach, Faroy, Ebstein, Kahana, \& Levine, 2001) as well as less exploratory behavior in 1-year-old infants (Ebstein et al., 1998). These data suggest that low dopamine is a marker of less optimal infant development and that prenatal maternal depression and low dopamine levels may be precursors to infants' developmental delays.

The relationship between dopamine and depression is complicated, however, by dopamine's interaction with several other neurotransmitters including serotonin and norepinephrine (Kalia, 2005). As several have noted, the norepinephrine, dopamine and serotonin systems have reciprocal interactions, making it difficult to act on a specific system without a cascade of events affecting the other two systems (Trivedi, 2006). One research group has reported an interaction between serotonin and dopamine (Esposito, 2006), and another study has documented an interaction between dopamine and norepinephrine (Nutt, 2006).

\footnotetext{
* Corresponding author at: Touch Research Institute, University of Miami Medical School, PO Box 016820, Miami, FL 33101, United States. Tel.: +1 3052436781 .

E-mail address: tfield@med.miami.edu (T. Field).
} 
Further, cortisol hypersecretion in depressed patients has been correlated with dopaminergic dysfunction (Duval et al., 2006). In this study, chronic elevation of cortisol led to dopaminergic and noradrenergic dysfunction. Others have reported that patients who had high plasma cortisol levels had low dopamine levels (Mitani, Shirayama, Yamada, \& Kawahara, 2006).

The purpose of the present study was to determine the effects of high prenatal dopamine levels on prenatal maternal depression and on other neurotransmitters during pregnancy and to assess neonatal biochemical and behavioral differences related to high prenatal dopamine levels. No literature could be found on the effects of dopamine levels on prenatal depression except the research already noted from our lab (Field et al., 2004). In that longitudinal follow-up of a large sample of prenatally depressed women, we noted elevated cortisol and norepinephrine and low levels of serotonin and dopamine. In turn, the biochemical profile noted in the prenatally depressed women was also evident in their neonates (Field et al., 2004).

To assess the effects of dopamine levels on prenatal depression and neonatal outcome, the pregnant women in the present study were divided into the top and bottom tertiles based on their dopamine levels at 20 weeks gestational age. The higher prenatal maternal dopamine group was expected to have higher prenatal serotonin and lower cortisol levels as well as lower depression scores. The clinical relevance of this study was the assessment of potential biochemical markers for negative pregnancy outcomes.

\section{Method}

\subsection{Participants}

The participants were 126 depressed pregnant women. The women averaged 25 years of age, their socioeconomic status was low to middle ( $M=3.3$ on the Hollingshead index), they were distributed $55 \%$ Hispanic, $35 \%$ Black and $10 \%$ White, and $30 \%$ were married. The high and low dopamine groups did not differ on these demographic variables.

\subsection{Procedure}

The pregnant women were recruited at prenatal clinics during their first ultrasound visit $(M=20$ weeks gestation, $R=16-22$ weeks). Following informed consent, the pregnant women were given the Structured Clinical Interview for Depression(SCID). Although illicit drug use was an exclusionary criterion, none of the women reported this problem when questioned about drug use during the background interview. The women were assessed at 20 weeks and again at 32 weeks gestation, and their infants were evaluated for biochemical and behavioral profiles at the neonatal period ( $M=2$ days). The assessments were made by research associates who were "blind" to the women's group assignment.

\subsubsection{Structured Clinical Interview for DSM-IV Disorders (SCID)}

All women in the study were given the research version of the SCID (more structured version) at the 20-week gestation period to determine depression and anxiety diagnoses and to screen out other disorders including bipolar disorder, schizophrenia and other psychotic disorders. The women were diagnosed with Dysthymia or Major Depression on the SCID based on DSM-IV symptoms. Both depression types (Major Depression and Dysthymia) and those diagnosed with co-morbid anxiety disorder were eligible for the study. The SCID was administered by research associates following training and with continuing on site supervision by a clinical psychologist. In our experience (including a recent survey sample), the majority of the depressed pregnant women who attend the university ultrasound clinic were not taking anti-depressants and were not receiving other treatments for depression.

\subsubsection{The Center for Epidemiological Studies-Depression Scale (CES-D)}

This is a 20-item scale that assesses the frequency of depressive symptoms within the last week (Radloff, 1977). With scores ranging from 0 to 60 , a cut-off score of 16 is used for classifying a major depressive episode. With only a $6 \%$ false positive and 36\% false negative rate (Myers \& Weissman, 1980), this scale has been shown to be reliable and valid for diverse demographic groups and has been successfully used as a self-report assessment of depression in a number of studies that involved similar populations (e.g., Diego et al., 2004). This scale was given at the 20 -week gestation period.

\subsubsection{Urinary catecholamines and metabolites}

Urinary samples were collected from the women at the beginning of the 20- and 32-week gestation visits at mid-morning and from the neonates within the first 2 days post-delivery. No systematic differences were noted in the sampling or the timing of collection of urine samples between women. Urine samples were transferred to plastic vials and frozen without using acid or other preservatives. Assays were conducted by high-pressure liquid chromatography (HPLC) with electrochemical detection (Kilts, Gooch, \& Knopes, 1984). Urinary levels of the dopamine metabolite, the serotonin metabolite, and the norepinephrine metabolite were measured, as the majority of excreted dopamine and serotonin respectively exist in the form of metabolites. Urinary cortisol was assayed in the stored urine samples by radioimmunoassay using a specific antiserum from Radioassay Systems Laboratories (Carson City, CA). 
Table 1

Means for self-report and biochemical variables for pregnant women divided into top and bottom (high and low) tertiles on prenatal dopamine (HVA) levels (S.D.s in parentheses)

\begin{tabular}{|c|c|c|c|c|}
\hline & Low dopamine & High dopamine & $F$ & $p$ \\
\hline \multicolumn{5}{|l|}{ Self-report variables } \\
\hline Depression (CES-D) & $19.6(10.4)$ & $16.3(10.0)$ & 4.01 & .05 \\
\hline Somatic-vegetative subscale (CES-D) & $9.5(2.1)$ & $6.3(1.5)$ & 5.05 & .05 \\
\hline \multicolumn{5}{|l|}{ Biochemical values (20 weeks GA) } \\
\hline Dopamine & $160.0(63.6)$ & $422.4(102.1)$ & 482.20 & .000 \\
\hline Serotonin & $3483.9(2041.4)$ & $4804.9(2389.7)$ & 13.92 & .000 \\
\hline Norepinephrine & $47.1(22.4)$ & $64.8(26.1)$ & 27.07 & .000 \\
\hline Cortisol & $230.8(153.0)$ & $238.3(104.8)$ & & NS \\
\hline \multicolumn{5}{|l|}{ Biochemical values ( 32 weeks GA) } \\
\hline Dopamine & $187.7(90.8)$ & $299.6(163.4)$ & 15.23 & .000 \\
\hline Serotonin & $2833.1(1730.9)$ & $3836.0(1978.6)$ & 4.60 & .04 \\
\hline Norepinephrine & $45.3(25.5)$ & $54.1(22.0)$ & & NS \\
\hline Cortisol & $267.3(136.1)$ & $277.6(133.3)$ & & NS \\
\hline
\end{tabular}

Table 2

Means for biochemical values and behavioral variables for neonates of women who were divided into top and bottom (high and low) tertiles on their prenatal dopamine (HVA) levels (S.D.s in parentheses)

\begin{tabular}{|c|c|c|c|c|}
\hline & Low dopamine & High dopamine & $F$ & $p$ \\
\hline \multicolumn{5}{|c|}{ Neonatal biochemical values } \\
\hline Dopamine & $278.3(141.7)$ & $690.1(193.9)$ & 126.44 & .00 \\
\hline Serotonin & $6464.9(4731.0)$ & $10469.6(6668.1)$ & 6.37 & .02 \\
\hline Norepinephrine & $62.4(36.3)$ & $73.5(47.9)$ & & NS \\
\hline Cortisol & $558.0(189.0)$ & $426.5(196.2)$ & 10.02 & .002 \\
\hline \multicolumn{5}{|c|}{ Neonatal behavioral variables } \\
\hline Autonomic Stability & $3.42(.57)$ & $4.75(.96)$ & 4.19 & .05 \\
\hline Excitability $^{\mathrm{a}}$ & $2.50(1.33)$ & $1.90(.93)$ & 4.86 & .05 \\
\hline
\end{tabular}

a Lower score is optimal.

\subsubsection{Brazelton Neonatal Behavior Assessment Scale}

The Brazelton Neonatal Behavior Assessment Scale (BNBAS) (Brazelton, 1978) was given midway between feedings. The scale is comprised of 20 neurological reflex items and 27 other items summarized according to 7 factors: habituation, orientation, motor behavior, range of state, state regulation, autonomic stability and abnormal reflexes. In addition, scores are given for excitability and depressive behaviors. The Brazelton examiners were unaware of the infant's group assignment and were trained to a .90 reliability criterion prior to the study.

\section{Results}

As can be seen in Table 1, ANOVA comparisons between high and low dopamine groups based on a tertile split yielded the following for the high $(N=42)$ versus low $(N=42)$ dopamine group: (1) lower CES-D depression and somatic-vegetative subscale scores (eating and sleep problems) at the 20-week gestational age period; (2) higher dopamine, serotonin and norepinephrine levels at the 20-week gestational age visit; (3) higher dopamine and serotonin levels at the 32-week gestational age visit. As can be seen in Table 2, the neonates of high dopamine mothers had: (1) higher dopamine and serotonin levels; (2) lower cortisol levels; (3) better scores on the autonomic stability and excitability subscales of the Brazelton Neonatal Behavior Assessment Scale, although the groups did not differ on the other Brazelton Subscales.

\section{Discussion}

These data are consistent with previous data on non-pregnant adults, showing that depression scores were higher in those with lower dopamine levels (Netter, 2006). A greater number of somatic/vegetative symptoms as reported by these pregnant women on the CES-D subscale are also consistent with the profile of anhedonia, apathy and dysphoria that has been associated with dopaminergic hypofunction (Bressan \& Crippa, 2005).

The simultaneously elevated serotonin in the pregnant women with elevated dopamine was consistent across pregnancy (20 and 32 weeks gestation), and is consistent with the interactive phenomena as reported by many (Esposito, 2006; Nutt, 2006; Trivedi, 2006). As Trivedi (2006) noted, the interactions of the dopamine, norepinephrine and serotonin systems suggest that one system affects the others, confounding the effects of each other. A similar interaction has been noted between cortisol and dopamine, with hypersecretion of cortisol being correlated with dopaminergic dysfunction (Duval et al., 2006; Mitani et al., 2006). Surprisingly, cortisol was not elevated in this group of pregnant women, although elevated cortisol was noted in the depressed pregnant women in our earlier study (Field et al., 2004). The neonates of high dopamine 
mothers had similarly higher dopamine and serotonin levels but also the expected lower cortisol levels based on the negative relationship noted between dopamine and cortisol in depressed adults (Duval et al., 2006; Mitani et al., 2006). The similarity between mothers' prenatal biochemical profiles and those of their neonates has been noted in other samples of depressed women by our group (Field et al., 2004; Lundy, Field, Cigales, Cuadra, \& Pickens, 1997).

That the infants of high dopamine mothers had better autonomic stability and less excitability may relate to their own dopamine levels being high. These findings are consistent with the data showing better temperament (less negative emotionality) in 2-month-old infants and less anger in 12-month-old-infants (during mild physical restraint) who have increased dopamine receptors (Auerbach et al., 2001). However, the absence of group differences on the Brazelton orientating subscale was surprising given that high dopamine infants have also been reported to have better orienting scores at 2 weeks and more exploratory behavior at 12-months (Ebstein et al., 1998). Finally, greater rates of learning (habituation) in infancy (at 3-months) and greater novelty seeking in adolescence have been associated with greater numbers of dopamine receptors (Laucht, Becker, \& Schmidt, 2006).

In summary, low prenatal maternal dopamine levels may be a marker for low dopamine levels in the neonate and a risk factor for later negative temperament and cognitive delays. These data suggest that prenatal urine samples could be assayed for these biochemical markers, and, together with CES-D scores, they could be used to identify at-risk pregnancies. Further research, however, is needed to assess these biochemical interactions and their longer-term effects as well as any interventions that might alter these profiles during pregnancy.

\section{Acknowledgments}

We would like to thank the mothers and infants who participated in this study. This research was supported by a Merit Award (MH \# 46586) and NIH grant (AT\# 00370) and Senior Research Scientist Awards (MH\#0033 1 and AT\# 001585) and a March of Dimes Grant (\# 12-FYO3-48) to Tiffany Field and funding from Johnson and Johnson Pediatric Institute to the Touch Research Institute.

\section{References}

Auerbach, J., Faroy, M., Ebstein, R., Kahana, M., \& Levine, J. (2001). The association of the dopamine D4 receptor gene (DRD4) and the serotonin transporter promoter gene (5-HTTLPR) with temperament in 12-month-old infants. Journal of Child Psychology and Psychiatry, 42, 777-783.

Brazelton, T. B. (1978). The Brazelton Neonatal Behavior Assessment Scale: Introduction. Monographs of the Society for Research in Child Development, 43, $1-13$.

Bressan, R. A., \& Crippa, J. (2005). The role of dopamine in reward and pleasure behavior-review of data from preclinical research. Acta Psychiatrica Scandinavica Supplement, 427, 14-21.

Dailly, E., Chenu, F., Renard, C., \& Bourin, M. (2004). Dopamine, depression and antidepressants. Fundamental and Clinical Pharmacology, 18, 601-607.

Diego, M., Field, T., Hernandez-Reif, M., Cullen, C., Schanberg, S., \& Kuhn, C. (2004). Prepartum, postpartum and chronic depression effects on newborns. Psychiatry, 67, 63-80.

Duval, F., Mokrani, M., Monreal-Ortiz, J., Fattah, S., Champeval, C., Schulz, P., et al. (2006). Cortisol hypersecretion in unipolar major depression with melancholic and psychotic features: Dopaminergic, noradrenergic and thyroid correlates. Psychoneuroendocrinology, 31, 876-888.

Ebstein, R., Levine, J., Geller, V., Auerbach, J., Gritsenko, I., \& Belmaker, R. (1998). Dopamine D4 receptor and serotonin transporter promoter in the determination of neonatal temperament. Molecular Psychiatry, 3, 238-246.

Esposito, E. (2006). Serotonin-dopamine interaction as a focus of novel antidepressant drugs. Current Drug Targets, 7, 177-185.

Field, T., Diego, M., Dieter, J., Hernandez-Reif, M., Schanberg, S., Kuhn, C., et al. (2004). Prenatal depression effects on the fetus and the newborn. Infant Behavior E Development, 27, 216-229.

Kalia, M. (2005). Neurobiological basis of depression: An update. Metabolism, 54, 24-27.

Kilts, C., Gooch, M., \& Knopes, K. (1984). Journal of Neuroscience and Methods, 11, 257-273.

Laucht, M., Becker, K., \& Schmidt, M. (2006). Visual exploratory behavior in infancy and novelty seeking in adolescence: Two developmentally specific phenotypes of DRD4? Journal of Child Psychology and Psychiatry, 47, 1143-1151.

Lundy, B., Field, T., Cigales, M., Cuadra, A., \& Pickens, J. (1997). Vocal and facial expression matching in infants of mothers with depressive symptoms. Infant Mental Health Journal, 18, 265-273.

Mitani, H., Shirayama, Y., Yamada, T., \& Kawahara, R. (2006). Plasma levels of homovanillic acid, 5-hydroxyindoleacetic acid and cortisol, and serotonin turnover in depressed patients. Program of Neuropsychopharmacology and Biological Psychiatry, 30, 531-534.

Myers, J., \& Weissman, M. (1980). Use of a self-report symptom scale to detect depression in a community sample. American Journal of Psychiatry, 137, $1081-1084$.

Netter, P. (2006). Dopamine challenges tests as an indicator of psychological traits. Human Psychopharmacology, 21, 91-99.

Nutt, D. (2006). The role of dopamine and norepinephrine in depression and antidepressant treatment. Journal of Clinical Psychiatry, 67, 3-8.

Radloff, L. (1977). The CES-D Scale: A self-report depression scale for research in the general population. Applied Psychology Measures, 3, $385-401$.

Trivedi, M. (2006). Major depressive disorder: Remission of associated symptoms. Journal of Clinical Psychiatry, 67, 27-32. 